\title{
Fabrication of Large-area 3-D Ordered Silver- coated Colloidal Crystals and Macroporous Silver Films Using Polystyrene Templates
}

\author{
Wen $\mathrm{Zhu}^{1,2}$, Yuanyuan $\mathrm{Wu}^{1}$, Chengyin Wang ${ }^{1, *}$, Ming Zhang ${ }^{1, *}$, Guanxiu Dong ${ }^{2}$
}

(Received 4 July 2013; accepted 12 August 2013; published online 9 September 2013)

\begin{abstract}
The highly ordered silver-coated colloidal crystals arrays and macroporous silver films were derived through an electrostatics-induced adsorption effect using polystyrene (PS) as templates. Carboxyl-modified PS microspheres were prepared by emulsifier-free emulsion polymerization using methacrylic acid (MAA) as the functional monomer. PS microspheres were self-assembled into close packing colloidal crystals of facecentered cubic arrays to the substrate with vertical deposition method. These colloidal crystals were modified using dopamine (DA) to form poly-dopamine (PDA) during its oxidative polymerization. Through electrostatic interaction, the silver nanoparticles were deposited and adsorbed onto the surfaces of colloidal crystals templates by exposing $\left[\mathrm{Ag}\left(\mathrm{NH}_{3}\right)_{2}\right]^{+}$solution to infrared irradiation. Removal of the polymeric template by etching with methylbenzene solvent resulted in 3D ordered macroporous silver films. The structural and properties of the ordered silver-coated arrays and macroporous silver films were characterized by field emission scanning electron microscopy (FE-SEM), X-ray diffraction (XRD), UV-vis spectroscopy and surface-enhanced Raman spectroscopy (SERS). The results indicate that the prepared silver-coated arrays and macroporous silver films possess the features of ordered multilayer arrangement, uniformity and repeatability as well as an ideal SERS effect.
\end{abstract}

Keywords: Polystyrene colloidal crystal; Template; Dopamine; Silver-coated array; Ordered macroporous film

Citation: Wen Zhu, Yuanyuan Wu, Chengyin Wang, Ming Zhang and Guanxiu Dong, "Fabrication of Largearea 3-D Ordered Silver-coated Colloidal Crystals and Macroporous Silver Films Using Polystyrene Templates", Nano-Micro Lett. 5(3), 182-190 (2013). http://dx.doi.org/10.5101/nml.v5i3.p182-190

\section{Introduction}

Noble metal nanostructures have recently been studied for their extraordinary surface plasmon resonance (SPR) phenomenon, which is a collective electron oscillation caused by electromagnetic $(\mathrm{EM})$ radiation and its movement along the interface between metals and dielectric materials [1-3]. Localized surface plasmon resonance excitations in noble metal nanoparticles, easily accessible with UV-visible light, determine their unique optical properties and result in a wide range of appli- cations of noble metal nanoparticles [4,5]. Therefore, highly ordered noble metal nanostructure arrays should be of interest for the development of optical waveguides, sub-diffractional microscopy, and bio-chemical sensors, because of guaranteeing the sensing devices employing SPR effects [6-8].

Several methods for the fabrication of metallic nanostructures have been reported, including the creation of ordered nanostructures based on templates by using the evaporation system or lithography/etching technique, which are either difficult to obtain consistency

\footnotetext{
${ }^{1}$ College of Chemistry and Chemical Engineering, Yangzhou University, Yangzhou City 225002, China

${ }^{2}$ College of Materials Science and Engineering, Jiangsu University of Technology, Changzhou City 213001, China

*Corresponding author. Email: lxyzhangm@yzu.edu.cn (M. Zhang), wangcy@yzu.edu.cn (C. Y. Wang).
} 
or complicated to require expensive equipments [9-12]. As early as 2000, Velev and Kaler used colloid crystalline templates to achieve a porous metals structure [13]. A decade later, Shimomura and co-workers employed a simple respective original structures of electroless plating to create silver-deposited honeycombpatterned films and pincushion films [14]. More recently, Lee and Haynes formed self-assembled periodic nanohole arrays over a millimeter-sized area of metallic film [15]. Directed self-assembly of functionalized nanoparticles, due to the possibility of controlling the spacing and organization in the resulting nanocomposite systems, has probably given the greatest diversity of architectures.

In this paper, we adopted an easy and inexpensive method to fabricate highly ordered silver-coated arrays and macroporous silver films. Mono-dispersed PS microspheres could be self-assembled into colloidal crystals of ordered three-dimensional structure by electrostatic repulsion $[16,17]$. The emulsifier-free emulsion polymerization was employed to produce monodispersed PS microspheres, and then by vertical deposition method, the PS microspheres were self-assembled into ordered colloidal crystals on glass substrates. We combined both the colloidal crystals, used as templates for the growth of silver nanoparticles, and the complexation of $\mathrm{Ag}^{+}$ions to form well-organized layers of dense silver nanoparticles with a narrow size distribution. The polydopamine with amine and catechol groups played an extremely important role by anchoring silver ions into the PS matrix [18]. The result was the formation of a self-organized arrangement of silver nanoparticles located just on the colloidal crystals surface, such as round-top hexagonal (RTH) nanostructures possessed characteristics similar to those of the fractals with regard to shape and size. After the polystyrene template was removed with chemical solvent, a well-ordered macroporous silver film in 3DOM was formed on the substrate. The experimental approach of this work was simple and easy to operate.

\section{Experimental}

\section{Materials}

Styrene (St) was purchased from Shanghai Chemical Reagent Co. (China) and purified by treating with $5 \% \mathrm{NaOH}$ aqueous solution to remove the inhibitor. Potassium persulfate $\left(\mathrm{K}_{2} \mathrm{~S}_{2} \mathrm{O}_{8}\right.$, KPS $)$ was purified by recrystallization in absolute ethanol (EtOH). Silver nitrate $\left(\mathrm{AgNO}_{3}, 99 \%\right)$, ammonia hydroxide $\left(\mathrm{NH}_{3} \cdot \mathrm{H}_{2} \mathrm{O}\right)$, methylbenzene and methacrylic acid (MAA) were also purchased from Shanghai Chemical Reagent Co. (China) and used as received. Dopamine (DA) and trihydroxymethyl aminomethane (Tris) were purchased from Aladdin Chemical Co. Limited. Deionized water was used for all the experimental processes.

\section{Fabrication of mono-dispersed PS microspheres and PS colloidal crystals}

The carboxyl-modified PS microspheres were synthesized by emulsifier-free emulsion polymerization using MAA as a functional comonomer. The typical recipe was as follows: $80.0 \mathrm{~g}$ deionized water, $0.15 \mathrm{~g}$ KPS, $1.1 \mathrm{~g}$ MAA and $11.0 \mathrm{~g}$ St were charged into four-neck round flask equipped with mechanical stirrer, $\mathrm{N}_{2}$ inlet, thermometer with temperature controller, and condenser. The vessel was immersed into a thermostat water bath. The reaction solution was deoxygenated by bubbling with nitrogen gas at room temperature for about $30 \mathrm{~min}$, and then followed by heating to $75^{\circ} \mathrm{C}$. Under a stirring rate of $300 \mathrm{rpm}$, the polymerization was continued for $6 \mathrm{~h}$ at constant temperature. By the tuning of the amount of KPS and MAA, carboxylmodified polystyrene with different particle size could be obtained and for further use.

Microscope slides were cut into about $1.0 \mathrm{~cm} \times 3.0$ $\mathrm{cm}$ pieces and were soaked in ethanol, acetone in sequence under ultrasonic cleaning, immersed into isopropanol for $30 \mathrm{~min}$, then rinsed with deionized water and dried by high purity nitrogen, using as substrates. Vertical convective self-assembly deposition was achieved to obtain high-quality colloidal crystals. Each cleaned substrate was dipped into the suspensions of mono-dispersed PS vertically in a vial. The suspensions were placed in a constant temperature at $45^{\circ} \mathrm{C}$ and humidity at $65 \% \mathrm{RH}$ until water was completely evaporated form the dispersions, adding a large beaker with holes outside the vial so as to reduce the influence of wind force to the self-assembly. The colloidal crystals were then dried naturally at room temperature for $24 \mathrm{~h}$.

\section{Preparation of ordered silver-coated arrays and macroporous silver films}

The PS colloidal crystals were immersed in the PDA solution (0.02 g dopamine, $0.12 \mathrm{~g}$ TRIS hydroxy methyl aminomethane and $10.0 \mathrm{~g}$ deionized water), and then were dried under the infrared lamp to form the PDAmodified colloidal crystal templates. The $\left[\mathrm{Ag}\left(\mathrm{NH}_{3}\right)_{2}\right]^{+}$ solution was prepared by adding ammonia water slowly into $\mathrm{AgNO}_{3}$ solution and stirring them constantly, until the muddy solution turned to transparent. The prepared PDA-modified PS colloidal crystal templates were immersed into $\left[\mathrm{Ag}\left(\mathrm{NH}_{3}\right)_{2}\right]^{+}$solution and then were dried keeping in the infrared lamp. The silver nanoparticles were deposited coating on the colloidal crystals. After the vacuum drying, the ordered silvercoated arrays were obtained. After removing colloidal crystal template by etching in methylbenzene solvent at room temperature for $24 \mathrm{~h}$, a well-ordered macroporous 
silver film in 3DOM was formed on the substrate.

\section{Characterization}

Field emission scanning electron microscopy (FESEM) and energy dispersion spectrum analysis (EDS) were carried out with a HITACHI S-4800 scanning electron microscope operating at an acceleration voltage of $15 \mathrm{kV}$. X-ray powder diffraction (XRD) measurements were performed on a BRUKER D8ADVANCE $\mathrm{X}$-ray diffract meter with $\mathrm{Cu}-\mathrm{K} \alpha$ radiation. The absorption spectra (UV-Vis) were collected on a VARIAN CARY 5000 spectrophotometer. Surface-enhanced Raman spectroscopy (SERS) was performed with a RENISHAW IN VIA Raman spectrometer.

\section{Results and Discussion}

\section{Effect of PDA on the formation of PS/Ag com- posite structures}

After the polymerization process, mono-dispersed PS microspheres with spherical shape were obtained as shown in Fig. 1(a). It can be seen clearly that the formed PS microspheres are of smooth surfaces, and the size distribution of individual microspheres is about $270 \mathrm{~nm}$ in diameter. Figure 1(b) shows the FESEM images of self-assembly colloidal crystal arrays from monodispersed PS spheres emulsion with a diameter of 270
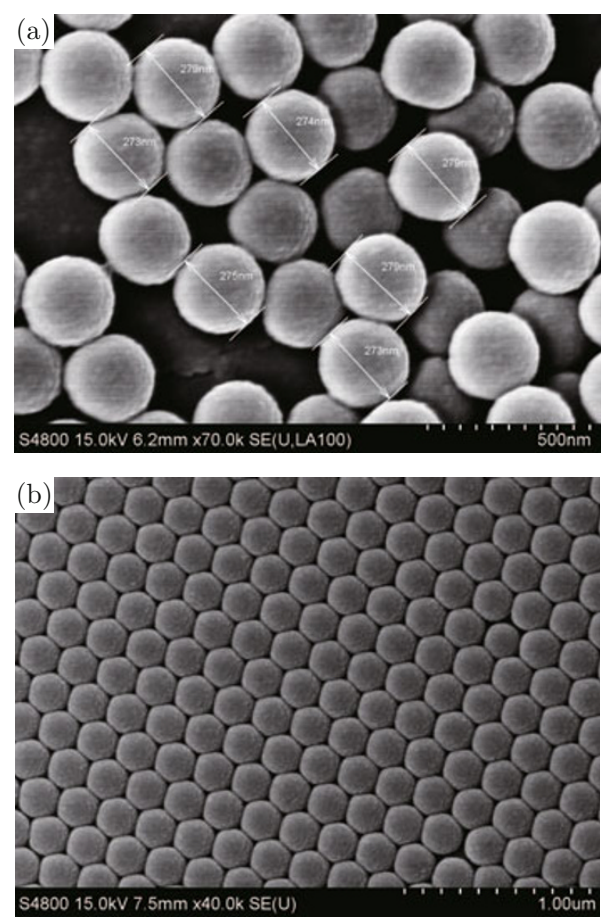

Fig. 1 FESEM images of (a) PS microspheres with a diameter of $270 \mathrm{~nm}$ synthesized by emulsifier-free emulsion polymerization and (b) PS colloidal crystal arrays assembled by vertical deposition. nm. Each PS microsphere is surrounded with six of them, forming the close-packed hexagonal structure with thermal stability and in a certain large area. The insert shows that, therefore, it can be used as a template but also existing some holed defects. During the self-assembly process, keeping a dilute concentration of colloidal crystals will get a strong interaction force between particles, and will contribute to the spontaneous crystallization assisted by electrostatic interaction in the deposition process $[19,20]$. Meanwhile, the modified microsphere surface with carboxyl group and the functioned hydrogen bond in carboxyl are to enhance the bonding strength between microspheres [21].

To our knowledge, few works had discussed in detail the mechanism of metal-doped composite microspheres' synthesis, the polymer beads were sensitized so that metal nanoparticle growth essentially started from the surface of each polymer bead [22]. Dopamine containing both amine and catechol functional groups could self-polymerize to form thin, surface-adherent polydopamine films onto a wide range of PS arrays [18]. The incorporation of PDA onto the surfaces of PS arrays was confirmed by FT-IR spectroscopy measurements. Curves 1 and 2 in Fig. 2 show FT-IR spectra of PS and PDA-coated PS, respectively. Compared with the IR spectrum of PS in curve 1, the presence of typical peak of the N-H bending vibration band $\left(1662 \mathrm{~cm}^{-1}\right)$ in curve 2 of PDA-coated PS confirmed the incorporation of PS and PDA.

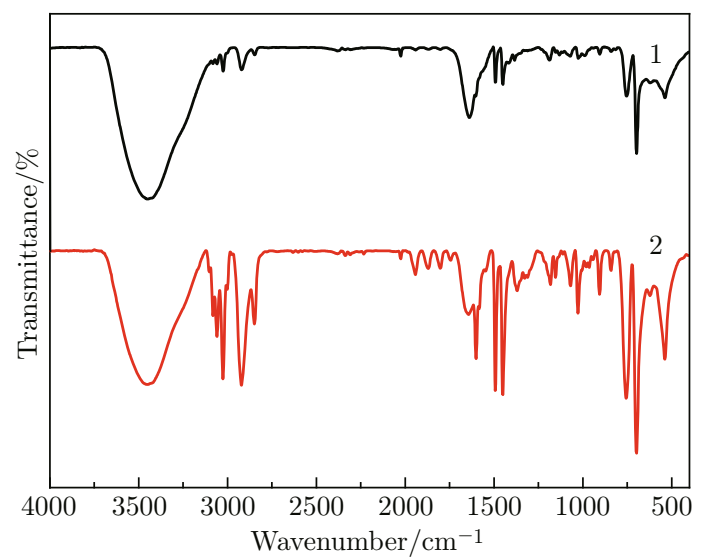

Fig. 2 FTIR spectra of PS (1) and PDA-coated PS (2).

PDA adheres to many types of surfaces, and the catechol group has been used as a linker between colloids and nanoparticles. The polydopamine coating affects the adherent and uniformity of metal deposition [18,23]. Figure 3 shows the FESEM images of the silver-coated array nanostructures prepared by without PDA and using PDA. As seen in Fig. 3(b), the surface of spheres becomes rough because a relatively uniform and dense coverage of silver nanoislands on the PS beads is also achieved. PDA improves the dispersion of hydrophobic 
PS microspheres in aqueous solution because of its hydrophilicity, and the metal-binding ability of phenolic hydroxyl and nitrogen-containing groups present in the PDA structure is exploited to absorb $\left[\mathrm{Ag}\left(\mathrm{NH}_{3}\right)_{2}\right]^{+}$complex ions onto the PDA-coated PS microspheres surface. The absorbed $\left[\mathrm{Ag}\left(\mathrm{NH}_{3}\right)_{2}\right]^{+}$complex ions are reduced to zero-valent silver by the reducibility of PDA, and silver nuclei are formed on PS microspheres surfaces. Nuclei are created at the silver ions bound to PDA-coated PS microspheres by the nucleation site role of $\mathrm{Ag}^{+}$ions bound to PDA, similar with the role of seed materials $[24,25]$.

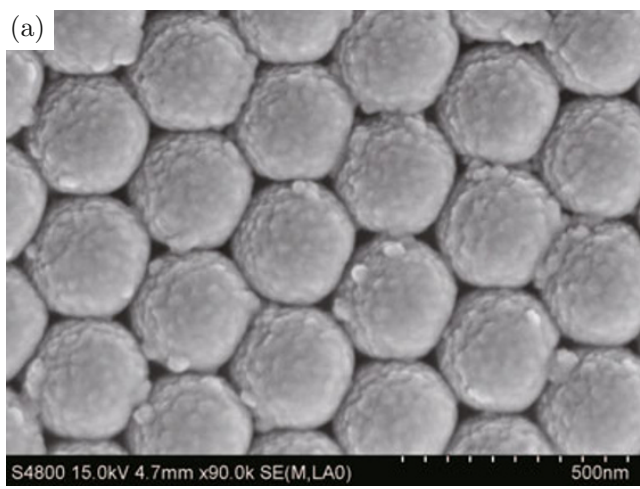

\section{Characterization of ordered silver-coated arrays and macroporous silver film}

Figure 4 shows the FESEM images of the resultant island silver films over close-packed arrays of dielectric polystyrene nanoparticles prepared by a combination of a seeded growth technique for metal coating on isolated colloids and the template-directed patterning methods. As seen in Fig. 4(a), the round-top hexagonal closedpacking of $270 \mathrm{~nm}$ polystyrene nanoparticles coated with silver nanoislands films are fabricated, which are highly reproducible because they imitate the bottom

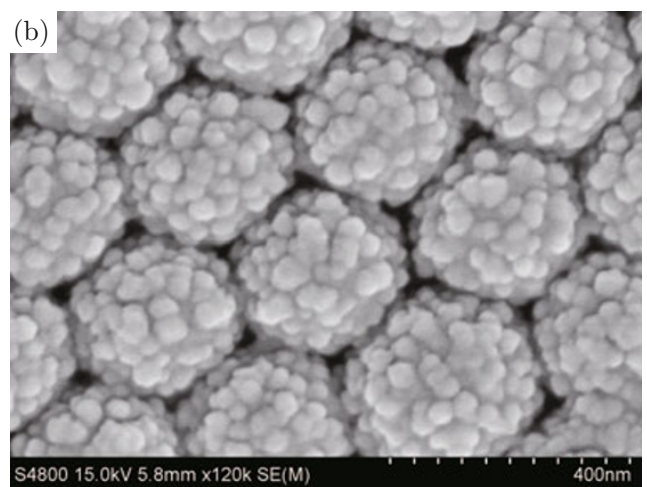

Fig. 3 FESEM images of (a) the silver-coated array nanostructures prepared by without PDA and (b) the silver-coated array nanostructures prepared by using PDA.
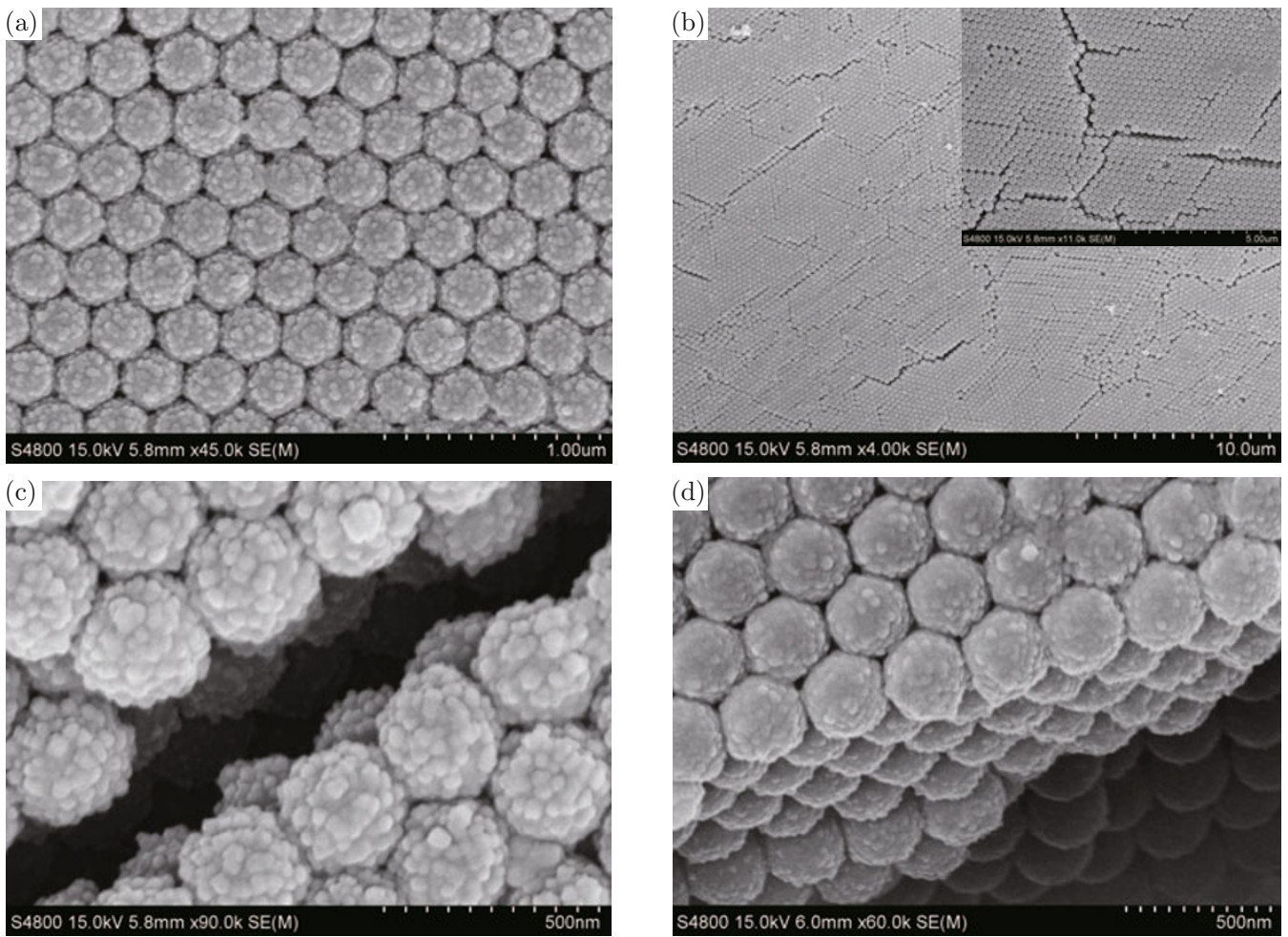

Fig. 4 FESEM images of the ordered silver-coated array nanostructures. (a) (b) Top view images; (c) (d) Cross-section images. 
shape of the PS colloidal crystal. The upper layer of the structure is analogous to silver films over nanostructures. According to capillary condensation, which is the adopted terminology to depict phase transformation in nanoconfined surfaces, conical shapes of metal arrays can be realized easily by using a nanoscale template $[10,26]$. From the inset of Fig. 4(b), few vacancies and the fracture lines in upper layer are found as the characteristic pattern. The long range ordered stacking in the obtained sample can be seen. Figure 4(c) and $4(d)$ show that the ordered silver-coated array films are three-dimensional nanostructures, and the number of multilayer arrangement is about 10 .

Figure 5(a) presents the energy spectrum analysis of the ordered PS/Ag arrays. The detection of Ag element in the spectrum shows that Ag particles have been successfully absorbed on the surface of colloidal crystal. The weight fraction of Ag element is $48.47 \%$, atom fraction being $9.48 \%$. The peak of carbon is attributed to PS substrate, and another peak is Au because that the sample is sputtered with a thin film of gold to increase the surface conductivity for FESEM. Figure 5(b) shows XRD spectrum of the same sample. The four diffractive peaks around $38.1^{\circ}, 44.3^{\circ}, 64.4^{\circ}, 77.4^{\circ}$ are characteristic peaks of $\mathrm{Ag}(111), \operatorname{Ag}(200), \operatorname{Ag}(220), \operatorname{Ag}(311)$, crystal plane of the face-centered cubic (fcc) structure respectively. Without obvious impurity peaks and similar to the standard diffractive spectrum of the pure silver (JCPDS card No 4-783), all above can prove that the synthesized silver nanoparticles on the surface of template is of high purity and stable in air without the interference of silver oxide, etc.
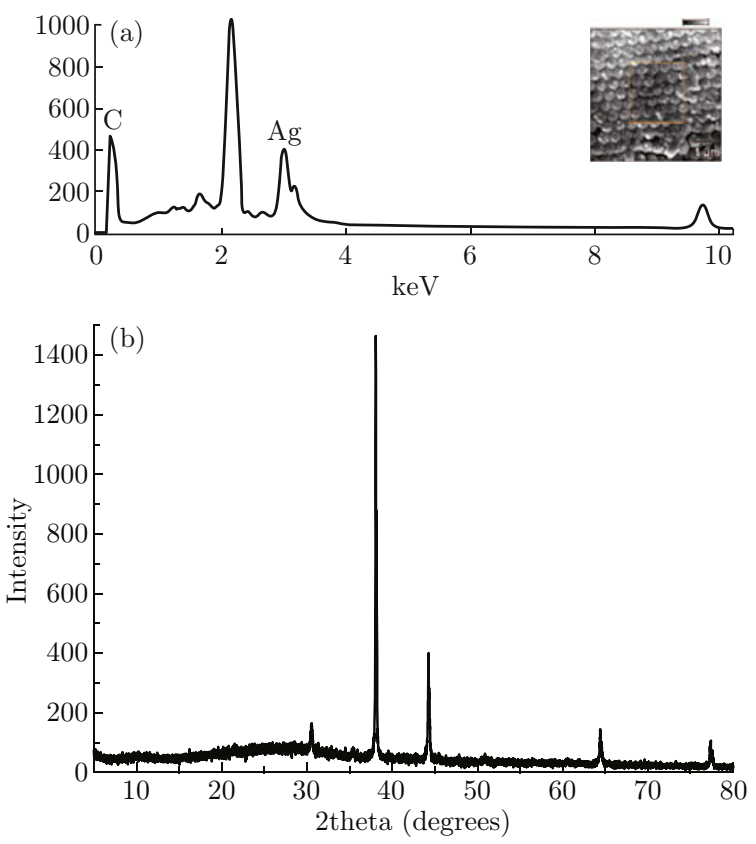

Fig. 5 (a) EDS analysis and (b) XRD pattern of the ordered silver-coated arrays.
A well-ordered silver macroporous structure can be fabricated by removing templates, as shown in Fig. 6 . Black holes connecting the neighboring pores are clearly observed in Fig. 6(a). Each hole is surrounded by other six holes. This structure is a hexagonal close-packed arrangement of spherical holes with visible three silver branches below each hole. Figure 6(b) shows that the average pore sizes are about $180 \pm 10 \mathrm{~nm}$, which results $30 \sim 35 \%$ shrinkage in comparison with the primary PS spheres.
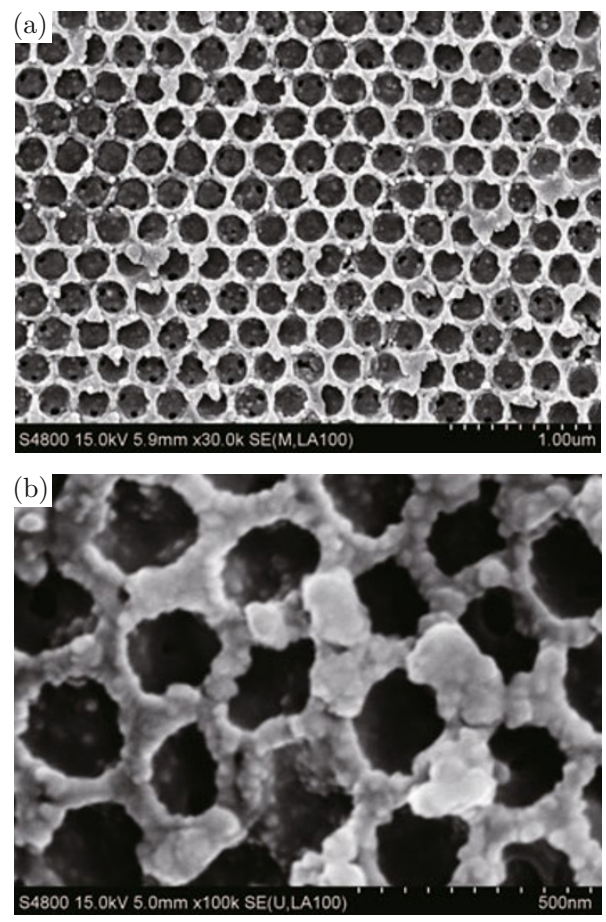

Fig. 6 Top view of FESEM images of the ordered silver macroporous structures.

\section{Optical properties analysis}

Highly ordered silver-coated arrays and porous films over several square centimeters in area can be further confirmed by UV-Vis spectra. The existence of stop-bands in the entire visible and near infrared regions is observed in the measurement of light absorption spectra, as shown in Fig. 7. The peak positions of absorbance are at about 639 and $646 \mathrm{~nm}$ for PS colloidal crystals and silver-coated arrays, respectively. The spectra from silver-coated arrays are shifted to longer wavelength compared to from colloidal crystals with an increased absolute absorption, demonstrating that the surface plasma resonance (SPR) peak of the silver nanocrystals in the colloidal crystals is found. Maybe it is due to plasmon coupling effect between silver particles closed to each other [27-30]. In addition, the hexagonal packing of the particles opens the potential for longer range dipole coupling [31]. The absorption band of the macroporous silver film is in the 
region of $420 \sim 460 \mathrm{~nm}$, depicting that the template diameters influence the optical property of ordered silver macroporous structures.

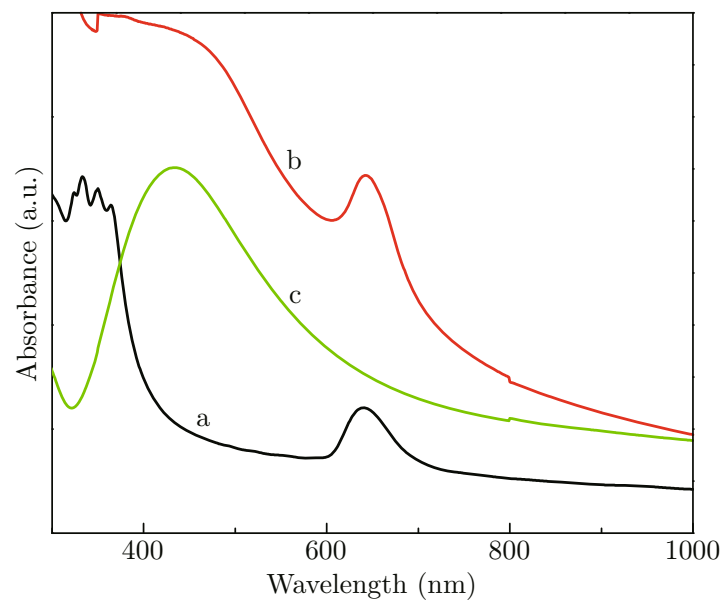

Fig. 7 UV-Vis absorption spectra of (a) PS colloidal crystals; (b) ordered silver-coated arrays; and (c) macroporous silver film, measured at a normal incidence of light.

Surface enhanced Raman scattering (SERS) is a type of abnormal optical enhanced effect based on the rough surface of nano-scale, particle morphology and the granular lumps, also it has been used as a highly sensitive analytical tool to study the interaction between molecule and the metal surface [32]. Theoretical model exhibits that the enhanced effect of metal particle polymer increases obviously compared with that of single particle [33]. It is of great use to expand the application of SERS technology when to fabricate and design highly ordered substrate of ideal Raman enhanced signal. In this study, SRES measurements are performed on the two distinct structures (ordered silver-coated arrays and macroporous silver film) for evaluation of their optical properties. Figure 8 presents the spectra of ordered silver-coated arrays and macroporous silver films dipped in the $1 \times 10^{-3} \mathrm{~mol} / \mathrm{l} \mathrm{R} 6 \mathrm{G}$ solution, almost all the distinctive peaks corresponde to the Raman lines for the R6G molecules. The observed peaks including $\nu(\mathrm{C}-\mathrm{H})$ out-of plane bend mode at $774 \mathrm{~cm}^{-1}, \nu(\mathrm{C}-$ C) stretching mode at $1360 \mathrm{~cm}^{-1}, 1508 \mathrm{~cm}^{-1}$ can be seen clearly in curve $\mathrm{b}$ and $\mathrm{c}$, which agree well with the literature [34]. For comparison, a silver flat film does not show any distinguishable enhanced Raman spectra. This evidence indicates that ordered silvercoated arrays and macroporous silver film as SERS substrates have excellent SERS performance. The enhancement factor measured from a macroporous silver film, $4.85 \times 10^{4}$, is significantly higher than $2.11 \times 10^{4}$ measured from a silver-coated arrays. However, the macroporous silver film shows a stronger enhancement of the SERS spectra than the ordered silver-coated arrays. As known from literatures, the SERS effects depend strongly on the roughness of the metal nanostruc- ture used as the substrate $[35,36]$. It is suspected that the reason can be attributed to the macroporous silver film surface roughness, in which the higher surface area can adsorb more R6G. The influence of concentration of R6G on SERS effects is studied and the SERS results are shown in Fig. 9. As for the $1 \times 10^{-3} \mathrm{~mol} / \mathrm{l}$ R6G without silver particles, the Raman lines for the R6G molecules can be seen from curve a where no peaks appeared. But when the $1 \times 10^{-6} \mathrm{~mol} / \mathrm{l}$ R6G deposits on the macroporous silver film, the peaks can be seen clearly. Curve b shows the SERS signals. With increasing of R6G to $1 \times 10^{-4} \mathrm{~mol} / \mathrm{l}$, it can be seen in curve $\mathrm{c}$ that the intensity of peaks becomes stronger. When the concentration of R6G increases further to $1 \times 10^{-3}$ $\mathrm{mol} / \mathrm{l}$, the Raman signals are magnified significantly as curve d shown. The macroporous silver film has a more affordable geometry to enhance electromagnetic field, providing lots of controllable "hotspots" [37,38].

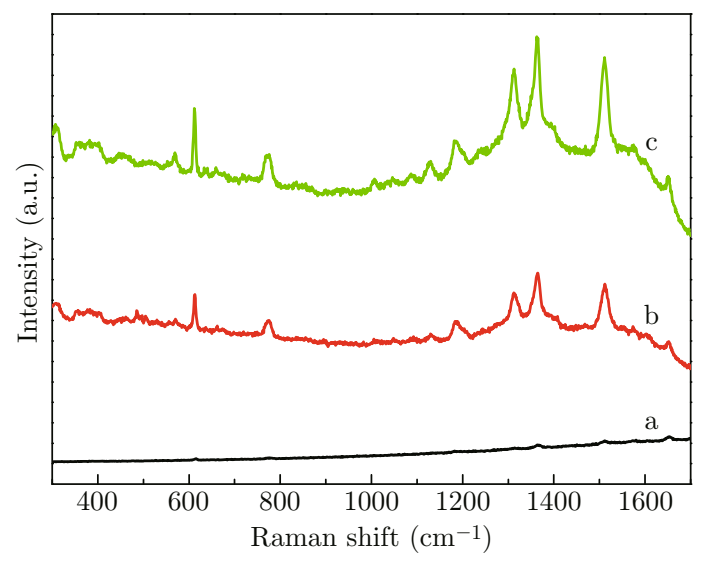

Fig. 8 SERS spectra of (a) $1 \times 10^{-3} \mathrm{~mol} / \mathrm{l}$ R6G on a silver flat film; (b) $1 \times 10^{-3} \mathrm{~mol} / \mathrm{l}$ R6G on a silver-coated array; and (c) $1 \times 10^{-3} \mathrm{~mol} / \mathrm{l} 6 \mathrm{G}$ on a macroporous silver film.

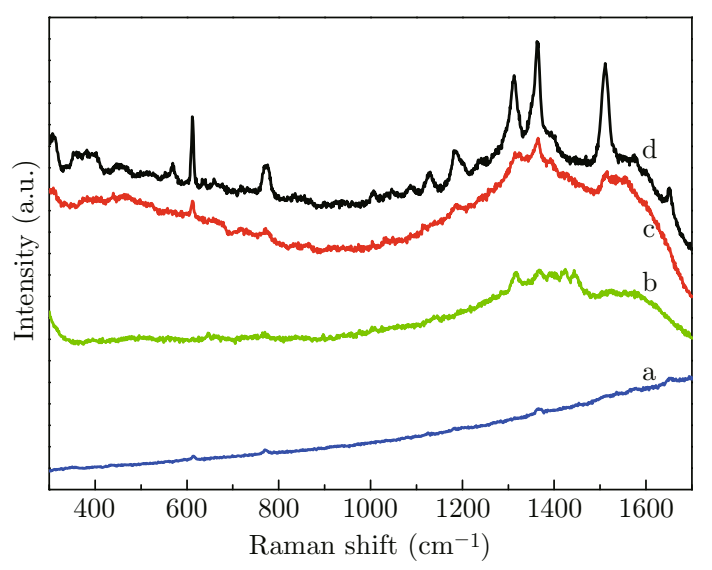

Fig. 9 Raman spectra of R6G. (a) $1 \times 10^{-3} \mathrm{~mol} / \mathrm{l}$ without silver particles; (b) $1 \times 10^{-6} \mathrm{~mol} / \mathrm{l}$ on a macroporous silver film; (c) $1 \times 10^{-4} \mathrm{~mol} / \mathrm{l}$ on a macroporous silver film; and (d) $1 \times 10^{-3} \mathrm{~mol} / \mathrm{l}$ on a macroporous silver film. 


\section{Conclusions}

Consequently, we successfully fabricated a regular hemispherical corrugation silver nanoislands film and macroporous silver film with polystyrene colloidal crystals as the template. The structural studies showed that three-dimensional highly ordered silver-coated arrays retained mainly the hexagonal closed-packing shape of the PS colloidal crystal, and the pore sizes of macroporous silver film depended on the diameter of polystyrene templates. The absorption spectra displayed a stronger high energy red-shifted peak for the ordered silver-coated arrays which indicated the adjustable optical quality. In addition, we observed that macroporous silver film had a stronger SERS enhancement by Raman spectroscopy depended on the roughness of the nanostructure. The method used in this paper could also be applied to the coating of other metals on the surface of PS colloidal crystal.

Integrating with the characteristic optical properties, periodic structure and the large specific surface area, the three-dimensional ordered metal-coated nanomaterials enjoy an abroad and attractive future application. Shi had pointed out that plasmonic microstructures consisting of a metallic film coated with a periodic dielectric structure can support two kinds of surface modes: surface plasmon polariton (SPP) modes bound at the metal-dielectric interface, and guided modes (GMs) bound at the periodic dielectric structure [39]. The formation of ordered assemblies of controlled shape and size nanoparticles appears to be one of the important issues.

\section{Acknowledgements}

The work was supported by the National Natural Science Foundation of China (Grant Nos. 50873085 and 21375116), a project funded by the Priority Academic Program Development of Jiangsu Higher Education Institutions (PAPD).

\section{References}

[1] K. A. Willets and R. P. Van Duyne, "Localized surface plasmon resonance spectroscopy and sensing", Annu. Rev. Phys. Chem. 58(1), 267297 (2007). http://dx.doi.org/10.1146/annurev. physchem.58.032806.104607

[2] A. M. Schwartxberg and J. Z. Zhang, "Novel optical properties and emerging applications of metal nanostructures", J. Phys. Chem. C 112(28), 10323-10337 (2008). http://dx.doi.org/10.1021/jp801770w

[3] C. H. Xu, B. Xie, Y. J. Liu, L. B. He and M. Han, "Optimizing surface-enhanced Raman scattering by template guided assembling of closely spaced silver nanocluster arrays", Eur. Phys. J. D 52(1-3),
111-114 (2009). http://dx.doi.org/10.1140/epjd/ e2009-00057-1

[4] R. C. Jin, Y. C. Cao, E. C. Hao, G. S. Mdtraux, G. C. Schatz and C. A. Mirkin, "Controlling anisotropic nanoparticle growth through plasmon excitation", Nature 425, 487-490 (2003). http://dx.doi.org/10. 1038 /nature 02020

[5] K. L. Kelly, E. C. Coronado, L. L. Zhao and G. C. Schatz, "The optical properties of metal nanoparticles: the influence of size, shape, and dielectric environment", J. Phys. Chem. B 107(3), 668-677 (2003). http://dx.doi.org/10.1021/jp026731y

[6] A. J. Haes, S. L. Zou, G. C. Schatz and R. P. Van Duyne, "Nanoscale optical biosensor: short range distance dependence of the localized surface plasmon resonance of noble metal nanoparticles", J. Phys. Chem. B 108(1), 109-116 (2004). http://dx.doi.org/10. 1021/jp0361327

[7] E. Hao, S. Y. Li, R. C. Bailey, S. L. Zou, G. C. Schatz and J. T. Hupp, "Optical properties of metal nanoshells", J. Phys. Chem. B 108(4), 1224-1229 (2004). http://dx.doi.org/10.1021/jp036301n

[8] E. M. Hicks, S. Zou, G. C. Schatz, K. G. Spears, R. P. Van Duyne, L. Gunnarsson, T. Rindzevicius, B. Kasemo and M. Kall, "Controlling plasmon line shapes through diffractive coupling in linear arrays of cylindrical nanoparticles fabricated by electron beam lithography", Nano Lett. 5(6), 1065-1070 (2005). http://dx. doi.org/10.1021/n10505492

[9] Y. Yang, M. Tanemura, Z. R. Huang, D. L. Jiang, Z. Y. Li, Y. P. Huang, G. Kawamura, K. Yamaguchi and M. Nogami, "Aligned gold nanoneedle arrays for surface-enhanced Raman scattering", Nanotechnology 21, 325701 (2010). http://dx.doi.org/10.1088/ 0957-4484/21/32/325701

[10] X. J. Cheng, S. C. Tjong, Q. Zhao and R. K. Y. Li, "Facile method to prepare monodispersed Ag/polystyrene composite microspheres and their properties", J. Polym. Sci. A Polym. Chem. 47, 4547-4554 (2009). http://dx.doi.org/10.1002/ pola. 23507

[11] C. Ruan, G. Eres, W. Wang, Z. Zhang and B. Gu, "Controlled fabrication of nanopillar arrays as active substrates for surface-enhanced Raman spectroscopy", Langmuir 23(10), 5757-5760 (2007). http://dx.doi. org/10.1021/la0636356

[12] Juha M. Kontio, Hannu Husu, Janne Simonen, Mikko J. Huttunen, Juha Tommila, Markus Pessa and Martti Kauranen, "Nanoimprint fabrication of gold nanocones with $\sim 10 \mathrm{~nm}$ tips for enhanced optical interactions", Opt. Lett. 34(13), 1979-1981 (2009). http://dx.doi. org/10.1364/OL.34.001979

[13] O. D. Velev and E. W. Kaler, "Structured porous materials via colloidal crystal templating: from inorganic oxides to metals", Adv. Mater. 12(7), 531-534 (2000). http://dx.doi.org/10. 1002/(SICI) 1521-4095(200004) 12: 7\$<\$531: : AID-ADMA531\$>\$3. 0. CO; 2-S

[14] H. Yabu, Y. Hirai and M. Shimomura, "Electroless plating of honeycomb and pincushion poly- 
mer films prepared by self-organization", Langmuir 22(23), 9760-9764 (2006). http://dx.doi.org/10. 1021/la062228r

[15] S. H. Lee, K. C. Bantz, N. C. Lindquist, S. H. Oh and C. L. Haynes, "Self-assembled plasmonic nanohole arrays", Langmuir 25(23), 13685-13693 (2009). http:// dx.doi.org/10.1021/la9020614

[16] F. Caruso, M. Spasova, A. Susha, M. Giersig and R. A. Caruso, "Magnetic nanocomposite particles and hollow spheres constructed by a sequential layering approach", Chem. Mater. 13(1), 109-116 (2001). http:// dx.doi.org/10.1021/cm001164h

[17] Y. N. Fu, Z. G. Jin, Z. F. Liu, Y. Liu and W. Li, "Selfassembly of colloidal crystals from polystyrene emulsion at elevated temperature by dip-drawing method", Mater. Lett. 62(27), 4286-4289 (2008). http://dx. doi.org/10.1016/j.matlet.2008.07.004

[18] H. Lee, S. M. Dellatore, W. M. Miller and P. B. Messersmith, "Mussel-inspired surface chemistry for multifunctional coatings", Science 318(5849), 426430 (2007). http://dx.doi.org/10.1126/science. 1147241

[19] T. Aslamazova and S. Bogdanova, "Polymer-monomer and polymer-polymer interactions and their effect on the stability of emulsifier-free acrylate latexes", Colloids and Surfaces A: Physicochem. Eng. Aspects 104(2), 147-155 (1995). http://dx.doi.org/10.1016/ 0927-7757 (95) 03263-8

[20] Z. Y. Zhong, Y. D. Yin, B. Gates and Y. N. $\mathrm{Xia}$, "Preparation of mesoscale hollow Spheres of $\mathrm{TiO}_{2}$ and $\mathrm{SnO}_{2}$ by templating against crystalline arrays of polystyrene beads", Adv. Mater. 12(3), 206-209 (2000). http://dx.doi.org/10. 1002/(SICI) 1521-4095(200002) 12: 3\$<\$206: : AID-ADMA206\$>\$3. 0. C0;2-5

[21] C. E. Reese, C. D. Guerrero, J. M. Weissman, K. Lee and S. A. Asher, "Synthesis of highly charged, monodisperse polystyrene colloidal particles for the fabrication of photonic crystals", J. Colloid Interf. Sci. 232(1), 76-80 (2000). http://dx.doi.org/10.1006/ jcis. 2000.7190

[22] Y. Kobayashi, V. Salgueirino-Maceira and L. M. LizMarzan, "Deposition of silver nanoparticles on silica spheres by pretreatment steps in electroless plating", Chem. Mater. 13(5), 1630-1633 (2001). http://dx. doi.org/10.1021/cm001240g

[23] Y. Saito, T. Kawano, M. Shimomura and H. Yabu, "Fabrication of mussel-inspired highly adhesive honeycomb films containing catechol groups and their applications for substrate-independent porous templates", Macromol. Rapid Comm. 34(8), 630-634 (2013). http://dx.doi.org/10.1002/marc. 201200839

[24] J. Zhang, J. Liu, S.Wang, P. Zhan, Z. Wang and N. Ming, "Facile methods to coat polystyrene and silica colloids with metal", Adv. Funct. Mater. 14(11), 1089-1096 (2004). http://dx.doi.org/10. 1002/adfm. 200400119

[25] Y. Kobayashi, Y. Tadaki, D. Nagao and M. Konno, "Deposition of gold nanoparticles on silica spheres by electroless metal plating technique", J. Colloid Interf. Sci. 283(2), 601-604 (2005). http://dx.doi.org/10. 1016/j·jcis. 2004.09.002

[26] A. V. Neimark, P. I. Ravikovitch and A. Vishnyakov, "Bridging scales from molecular simulations to classical thermodynamics: density functional theory of capillary condensation in nanopores", J. Phys. Condens Matter 15(3), 347-365 (2003). http://dx.doi. org/10.1088/0953-8984/15/3/303

[27] X. C. Yang, H. X. Liu, L. L. Li, M. Huang and J. F. Zhao, "Review on influence factors of surface plasmon resonance for nobel metal nanoparticles", J. Funct. Mater. 2(41), 341-349 (2010).

[28] Y. Lu, Y. D. Yin, Z. Y. Li and Y. N. Xia, "Synthesis and self-assembly of $\mathrm{Au} @ \mathrm{SiO}_{2}$ core-shell colloids", Nano Lett. 2(7), 785-788 (2002). http://dx.doi.org/ 10.1021/nl025598i

[29] Y. G. Sun, S. Matsubara, M. Nogami, J. Shi and W. Huang, "One-dimensional self-assembly of gold nanoparticles for tunable surface plasmon resonance properties", Nanotechnology 17(11), 2821-2827 (2006). http://dx.doi.org/10.1088/0957-4484/17/11/015

[30] B. Guo, J. S. Luo, Y. J. Tang and J. P. Cheng, "Preparation of triangular silver nanoplates by seed-mediated growth and their characterization", High Power Laser and Particle Beams 19(8), 1291-1294 (2007).

[31] A. I. Maaroof, J. V. Nygaard and D. S. Sutherland, "Plasmon hybridization in silver nanoislands as semishells arrays coupled to a thin metallic film", Plasmonics 6(2), 419-425 (2011). http://dx.doi.org/10. 1007/s11468-011-9220-9

[32] J. F. Li, Y. F. Huang and Y. Ding, "Shell-isolated nanoparticle-enhanced Raman spectroscopy", Nature 464(7287), 392-395 (2010). http://dx.doi.org/10. 1038/nature08907

[33] E. Hao and G. C. Schatz, "Electromagnetic fields around silver nanoparticles and dimers", J. Chem. Phys. 120(1), 357-366 (2004). http://dx. doi.org/10. 1063/1.1629280

[34] P. Hildebrandt and M. Stockburger, "Surfaceenhanced resonance Raman spectroscopy of rhodamine 6G adsorbed on colloidal silver", J. Phys. Chem. 88(24), 5935-5944 (1984). http://dx.doi.org/10. 1021/j150668a038

[35] L. H. Qian, X. Q. Yan, T. Fujita, A. Inoue and M. W. Chen, "Surface enhanced Raman scattering of nanoporous gold: smaller pore sizes stronger enhancements", Appl. Phys. Lett. 90(15), 153120-153123 (2007). http://dx.doi.org/10.1063/1.2722199

[36] X. Y. Lang, P. F. Guan, L. Zhang, T. Fujita and M. W. Chen, "Size dependence of molecular fluorescence enhancement of nanoporous gold", Appl. Phys. Lett. 96(7), 073701 (2010). http://dx.doi.org/10.1063/ 1.3323104

[37] J. M. Kontio, J. Simonen, J. Tommila and M. Pessa, "Arrays of metallic nanocones fabricated by UV-nanoimprint lithography", Microelectron. Eng. 87(9), 1711-1715 (2010). http://dx.doi.org/10. $1016 / j$.mee. 2009.08 .015 
[38] L. Kong, R. Dong, H. Ma and J. C. Hao, "Au NP honeycomb-patterned films with controllable pore size and their surface-enhanced Raman scattering", Langmuir 29(13), 4235-4241 (2013). http://dx.doi.org/ $10.1021 /$ la305143v
[39] L. Shi, X. H. Liu, H. W. Yin and J. Zi, "Optical response of a flat metallic surface coated with a monolayer array of latex spheres", Phys. Lett. A 374(8), 1059-1062 (2010). http://dx.doi.org/10. $1016 / \mathrm{j} \cdot$ physleta.2009.12.033 